\title{
Anterior crossbite: What it truly reflects?
}

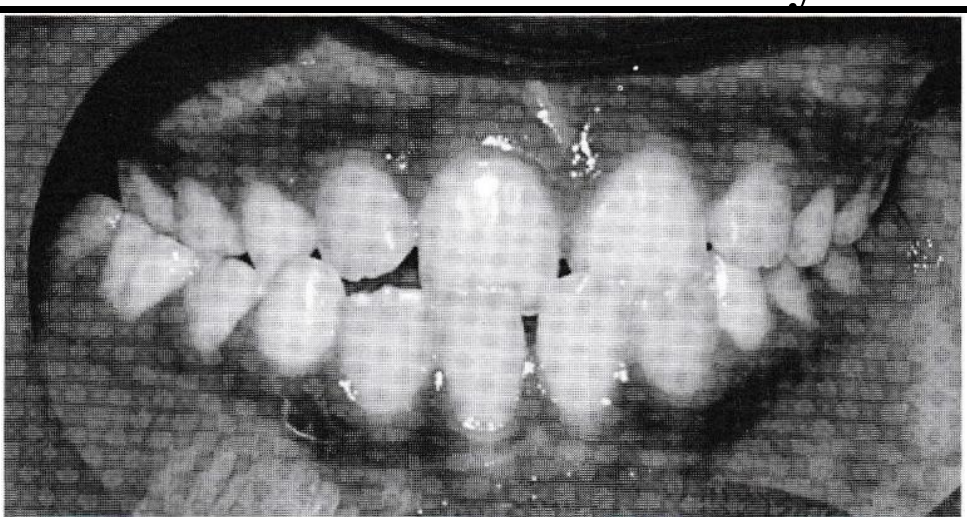

\section{Introduction}

Crossbite is a discrepancy in buccolingual relationship of the upper and lower teeth. When the labial surface of the lower anterior teeth occlude labial to the labial surface of the upper anterior teeth is called as anterior cross bite. The aetiology for anterior crossbite can be vary. Crossbite of all of the incisors is usually rarely found in children who do not have class III skeletal jaw relationship. A crossbite relationship of one or two anterior teeth, however, may develop in a child who has good facial proportions. A comprehensive understanding of the problem by the practitioner is a key element for successful management.

\section{The aetiology for anterior crossbite.}

1. Skeletal

Class III skeletal pattern (mild/moderate/severe)

- Genetics

- Cleft lip and palate patients.

- Syndromes - Cleidocranial dysplasia

Achondroplasia

Crouzon syndrome

Apert syndrome

- Endocrine problems - Acromegaly

- Trauma/ Fractures

2. Dental

Crossbite affecting only one or two teeth almost always is due to lingually displaced maxillary central or lateral incisors and this could be due to; (Figure 1)

- Crowding

- Supernumerary teeth/ Odontomes.

- Early loss of primary teeth. - Over retained deciduous teeth.

- Trauma

\section{Functional}

Displacement in order to obtain posterior occlusion due to edge-to edge incisor contact or to unsatisfactory transverse buccal segment relationship.

Dr. H.G.R.N Weerasinghe.

Senior Registrar in Orthodontics,

National Dental Hospital (Teaching) 
As aetiology of anterior crossbites is not merely dentoalveolar, correct diagnosis is mandatory to implement correct treatment protocol and there are several factors to be considered during patient assessment.

Factors to be considered in assessing a patient with anterior crossbite to arrive at a diagnosis.

\section{History}

- Age

- Past medical history.

- Past dental history.

- Family history.

- Habit history.

- Trauma history.

Clinical examination

Skeletal

- Antero-posterior, vertical and transverse relationship. Dental

- General dental condition- caries, periodontal condition (gingival recession in relation to the cross bite)

- Teeth present.

- Upper and lower arch alignment. (crowding, spacing and rotations)

- Buccal occlusion (canine and molar relationship)

- Overjet and overbite Soft tissues

- Facial aesthetics and profile. Functional

- Displacement of mandibular path of closure.

- Temporomandibular joint (TMJ) assessment.

$\underline{\text { Investigation findings }}$

- Plain radiographs - Intra oral- Presence of supernumerary teeth.

Extra oral- Fractures.

- Dental panoramic tomogram - Developmental stage of permanent teeth.

other pathologies, TMJ, asymmetry.

- Cephalometric radiographs - Lateral - Skeletal pattern.

Upper and lower incisor inclination (signs of dentoalveolar compensation.) Soft tissue profile.

Features of anterior crossbite without skeletal involvement.

- Identifiable local factor leading to lingual eruption of the incisors.

- Average skeletal pattern and soft tissue profile.

- Class I molar and canine relationship 


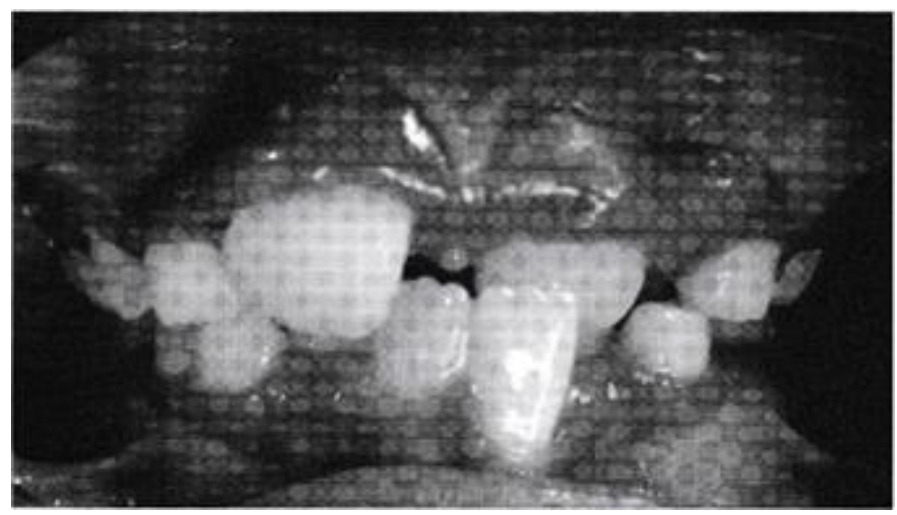

Figure 1: patient with anterior dental Crossbite

\section{Features of anterior crossbite with skeletal involvement.}

- No identifiable local aetiological factor.

- Presence of a family history. • Usually class III skeletal base with maxillary retrusion, mandibular prognathism or combination.

- Reduced cranial base angle which leads to forward positioning of the mandible.

- Normal or reduced maxillary mandibular plane angle and reduced lower face height. . Presence of skeletal asymmetry.

- Class III molar relationship.

- Dento- alveolar compensation. (Proclined maxillary incisors and retroclined mandibular incisors.)

\section{Anterior cross bite due to functional displacement.}

- Presence of a premature contact.

- Average skeletal, dental and soft tissue features at centric relation.

Anterior crossbites of skeletal in origin need combined orthodontic and surgical management. Some of the mild cases may respond to growth modification if addressed in correct time but those with excessive mandibular growth probably will require surgery for correction.

As knowing the patients' growth status is important in managing following methods can be used to assess the growth.

- Height charts.

- Weight charts.

- Comparison with parents and siblings.

- Development of secondary sexual characteristics.

- Cervical vertebral maturation stage. (Baccetti et al 2005).

- Hand wrist radiograph.

\section{Treatment options for anterior crossbite.}

Anterior crossbite without skeletal involvement.

\section{Importance of early treatment}

- Lingually positioned incisors limit lateral jaw movement.

- May get incisal attrition.

- Risk of developing gingival recession when the oral hygiene is less than the ideal. 
This requires opening enough space and moving the displaced tooth or teeth across the occlusion. It may be necessary to temporarily to separate the posterior teeth and create the vertical space to allow teeth to move. But during rapid growth in early adolescence this can be corrected without temporarily opening the bite.

* Removable appliance therapy.

- Double cantilever springs for facial movement of the maxillary teeth.

- Active labial bow for lingual movement of mandibular teeth. *

Fixed appliance therapy.

- Maxillary palatal arch with finger spring. (whip spring).

- Anterior cross elastics.

- 2 x4 appliance.

Anterior crossbite with skeletal involvement. 1.

\section{Early treatment}

Importance of early treatment

- To prevent progressive irreversible soft tissue or bony changes.

- To improve skeletal discrepancies and provide a more favorable environment for future growth.

- To improve occlusal function (prevent centric occlusion/ centric relation discrepancies)

- To simplify phase II comprehensive treatment. (mild to moderate class III may eliminate further surgical needs)

\section{Methods}

Orthopedic treatment.

- Reverse-Pull Headgear with or without rapid maxillary expansion to dental anchorage or traction to skeletal anchorage. (Figure 2\&3)

- Class III elastics to maxillary and mandibular plates.

- Chin caps.

Functional appliances - Frankel 3 appliance

Reverse twin block appliance.

Ideal patient for early treatment.

According to Turpin et al 1981;

- Good facial esthetics.

- Mild skeletal disharmony (ANB <-2 ${ }^{\circ}$ )

- No familial prognathism.

- With antero-posterior functional displacement.

- Symmetrical condylar growth

- Young patients with remaining growth potential. (Pre pubertal age 810years)

- Good cooperation. 

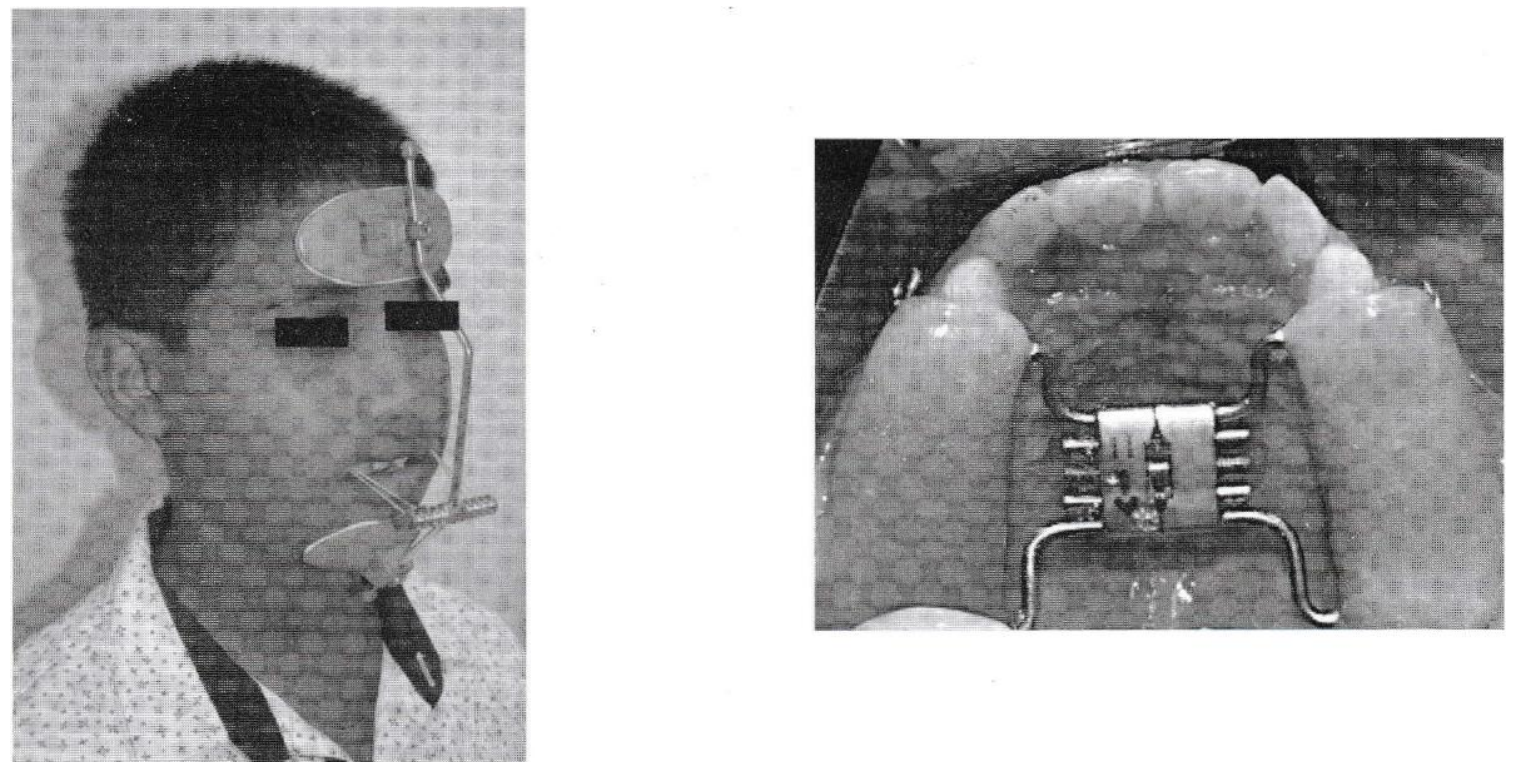

Figure 2: Early orthopedic correction with protraction headgear with rapid maxillary expansion.
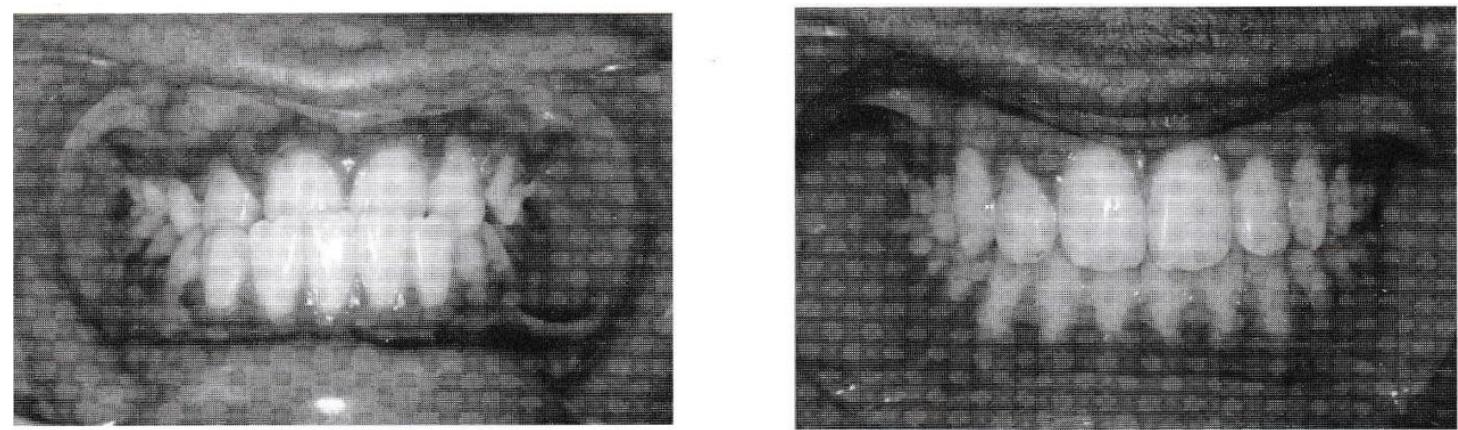

Figure 3: Pre and post treatment occlusion of a patient treated with facemask.

\section{Late treatment}

2.1. Orthodontic Camouflage.

Favorable features for orthodontics alone.

- No dental compensation. - Mild skeletal discrepancy.

- No remaining growth potential.

- Patient is able to bring incisors in to edge to edge position in centric relation.

- Low SNB or normal SNB angles.

- Low SNA warranting forward movement of the A point.

- Average soft tissue profile.

- Reduced lower face height.

2.2. Bone anchored maxillary protraction.

2.3. Orthodontic decompensation and orthognathic surgery.

When too severe for orthodontic camouflage and no remaining growth potential for orthopedic correction. 


\section{Conclusions}

Presence of an anterior Crossbite can be due to several reasons and correct diagnosis and timely management is very important. Simple dent alveolar cross bite should be treated early to prevent unnecessary soft tissue and hard tissue damage, prevent development of complicate malocclusion and expensive treatment. Early diagnosis of patients with skeletal involvement is beneficial as protraction in combination with expansion, if done in properly selected cases, may provide significant skeletal effects and alleviates the need for surgical intervention. Moreover, adult patients should be reassured and acknowledged on the availability of advanced orthodontic and orthognathic surgical options. Therefore, early diagnosis and timely referral for specialized care is a paramount.

\section{References}

1. Proffit RW. Contemporary Orthodontics. Sixth edition, Mosby 2019;13: 430-53.

2.Guyer EC, Ellis EE 3rd, McNamara JA Jr, Behrents RG. Components of class III malocclusion in juvenile and adoloscents. Angle orthod $1986 ; 56: 7-30$.

3.Roberston NRE. An examination of treatment changes in children treated with the fuctional regulator of Frankel. Am J Orthod 1983 113:204-12

4. Sugawara J, Asano T, Endo N, Mitani H. Long term effects of chincap therapy on skleletal profile in mandibular prognathism. J Orthod Dentofacial Orthop 1990; 98:12733.

5. Mandall N, DiBiase A, Littlewood S, Nute S, Stivaros N, McDowall R et al. Is early class III protraction facemask treatment effective' A multicenter, randomized controlled trial: 15 months follow up. J Orthod 2010; 37:149.61.

6. Vaughn GA, Manson B, Moon HB. The effects of maxillary protraction therapy with or without rapid palatal expansion: a prospective, randomized clinical trial. Am J Orthod 2005; 128: 299-309.

7. Mc Namara JA Jr. An orthopedic approach to the treatment of class III malocclusion in young patients. J Clin Orthod 1987; $21: 598608$.

8. Reynolds IR. The anterior cross bite: a simple method of treatment. B Dent J 1978; 144:143.46.

9. Kim JH. The effectiveness of facemask therapy: A meta-analysis. Am J Orthod Dento facial Orthop. 1999; 115:675-85.

10.Houston WJ. The current status of facial growth prediction: a review. B J Orthod 1979; 6:11-17. 